\title{
Can Metaphysical Structuralism solve the Plurality Problem?
}

\begin{abstract}
Metaphysics has a problem with plurality: in many areas of discourse, there are too many good theories, rather than just one. This embarrassment of riches is a particular problem for metaphysical realists who want metaphysics to tell us the way the world is and for whom one theory is the correct one. A recent suggestion is that we can treat the different theories as being functionally or explanatorily equivalent to each other, even though they differ in content. The aim of this paper is to explore whether the notion of functionally equivalent theories can be extended and utilised in the defence of metaphysical realism, drawing upon themes from structuralism in the philosophies of mathematics and science in which the specifics of theories do not matter as long as the relations in which they stand to other theories are maintained. I argue that despite its initial attractiveness, there are significant difficulties with this proposal. Discovering these obstacles (most probably) thwarts the realist structuralist project, but reveals interesting features of metaphysical systems.
\end{abstract}




\section{Can Metaphysical Structuralism solve the Plurality Problem?}

It is commonly, although not universally, acknowledged that there is a plurality problem in metaphysics; that is to say that in several areas of metaphysical enquiry there is more than one ontological theory which explains the phenomenon or phenomena under consideration acceptably well, not just in the eyes of its proponents but also in the opinion of those who would prefer an alternative ontology. These different theories often postulate different categories of entities and processes, invoke different primitives and rely upon different primitive presuppositions and yet they seem, on some level or other, to be doing the same explanatory job; or, to put the point slightly differently, the theories appear to be performing the same metaphysical function with different content.

The debate which has followed this observation has focused on various issues, such as whether plurality of metaphysical theories is a general problem in metaphysics or only concerns certain theories, and what the examples of plurality show about the entities postulated by the affected metaphysical theories and the interpretation of metaphysics in general. ${ }^{1}$ For the metaphysical realist, the pressing problems concern whether there is any way to determine which of the alternative theories (if any) is the true one or whether all of them are, and, if we can not determine which theory is correct, what the best attitude to metaphysics should be.

In this paper, I will concentrate upon the plausibility of just one realist response to the plurality problem: that we can maintain realism despite the plurality of metaphysical theories available by adopting a form of structuralism. This is, I will suggest, a natural extension of recent work which argues that some metaphysical theories are equivalent to each other relative to a specific metaphysical question (Miller 2005b; Benovsky 2008, 2016; Allen 2012) and aims to generalise from that position. This strategy adopts an attitude towards metaphysical disagreements similar to that which has been proposed to defuse seemingly intractable ontological disagreements in the philosophy of mathematics or difficulties for realism brought on by theory change in the philosophy of science (Benacerraf 1965, 1973; Worrall 1989; Ladyman et al. 2007). Perhaps the categories of entities which theories postulate, or even metaphysical theories themselves, are ontologically significant only in terms of the relations in which they stand to other categories and theories. If this is the case, what matters to metaphysics is structure and not the nature of the entities

1 These discussions are too wide-ranging to be covered in detail here. See, for instance, Chalmers et al. 2009; Tahko 2015. 
and primitive relations between them which are postulated by specific theories. If successful, this project would permit the reconciliation of metaphysical realism with the plurality of theories on offer; the structure, but not the entities and theories it relates, is real.

However, although I think that metaphysical structuralism is an option worth exploring, I will argue that ultimately it is an unconvincing way to defend realism. Despite this outcome, its failure illuminates some interesting features of metaphysical theorising and the connections between theories, in addition to narrowing down the options for realist construals of metaphysics.

I will begin by briefly sketching some examples of plurality in section 1 before considering what is meant by equivalence of metaphysical theories. This discussion reveals some important differences between metaphysicians with respect to both evidence and method, as well as disagreements about what counts as equivalence between theories. Having noted these and how they constrain putative equivalence claims, I will move on to investigate whether metaphysical structuralism is viable when theories are locally equivalent. I will suggest four reasons why it is not.

\section{Plurality in Metaphysics}

Examples of plurality abound in the literature - some more controversial than others - and so I shall mention just a few for the reader who is unfamiliar with the problem. But in this article I will not rigorously argue that these are in fact cases in which multiple metaphysical theories explain the same phenomenon or phenomena. These arguments have already appeared elsewhere and I will take that to be sufficient for the purposes of this discussion. In the next section I will also explore some reasons why philosophers have differing opinions about whether there are cases of plurality or not.

First, theories of objective similarity and difference can be framed in terms of universals (transcendent or immanent), different theories of tropes, resemblance classes or theories of primitive properties. There is good reason to think that many mature versions of these ontological theories are equally good at explaining what makes distinct particulars the same or different (or at least appear to be so), despite the different ontological categories and associated metaphysical apparatus which they employ. ${ }^{2}$

2 The restriction to 'mature' theories is important, since early versions of theories may be deficient in various ways which can be remedied. See Allen 2016 for a survey of these theories of properties; an argument that some of them are equivalent is made by Benovsky $(2008,2013)$ and the claim that the differences between them are not ontologically significant appears in Hirsch 1993. Even Armstrong (1997, 167-8) concedes that theories of tropes and of immanent universals have little to choose between them. 
Second, mereological theories of composition give varying accounts of when many things make up one: the nihilists say that they never do, universalists assert that every collection of simples composes a novel entity, while some theories attempt to give an account in which only some collections compose novel objects and others do not. On the latter theory, the collection of atoms currently asleep on my sofa composes a cat, while the collection of atoms which includes my cat's front left paw, the pizza in my fridge and Jupiter's moon Ganymede do not. Despite the differences in the claims which these theories make about what there is in the world, they each provide an explanation of the same intuitive and scientific data by which we attempt to measure the worth of our metaphysical theories, as well countering or explaining away the objections presented against them. Thus, for instance, the nihilists explain away the appearance of composite objects (Rosen and Dorr 2002), while the universalists attempt to explain why some composite objects seem so much more important than others. ${ }^{3}$

A third example of prima facie plurality is to be found among the different theories which explain the relationship between properties and laws of nature: in some theories, dispositional properties determine causal or nomic relations and thereby ground the existence of general causal laws; while according to other theories, the laws are ontologically more primitive than properties (and perhaps also more primitive than the things which instantiate the properties). ${ }^{4}$ The 'properties first' view includes various forms of dispositionalism about properties, while the 'laws first' view includes ontic structural realism (in its various forms). In other theories, neither laws nor properties are ontologically prior, such as in the Dretske-Tooley-Armstrong account of laws as necessary second order relations between universals. Although these theories disagree about what grounds what, they agree about the range of ontological categories which exist and what, in combination with each other, these categories do. Each theory provides an account of causal or nomic relations and objective similarity and difference. ${ }^{5}$

A fourth example is given by Kristie Miller (2005a), Eli Hirsch (2005, 2009) and McCall and Lowe (2006) who argue that there is nothing to choose (except perhaps terminology ${ }^{6}$ ) between endurantist and perdurantist theories of persistence. It is plausible to think of 3-dimensional entities

3 See Bennett (2009) for discussion of this case.

4 See, for instance, Allen 2012, Chakravartty 2017. There are of course alternative accounts of laws and properties, such as the broadly Humean Best Systems Analysis, which do not obviously appear to be equivalent.

5 In some sense, seeing the prima facie equivalence between these versions of properties and laws requires, to some extent, accepting the equivalence of the various ontological accounts of properties discussed previously. It is precisely this kind of combination of equivalent metaphysical theories which will be the topic of this article.

6 Although I use some of Eli Hirsch's examples, I do not think that the ontological realist gains by treating ontological disputes as disagreements about terminology. I will save a critique of Hirsch's position another occasion, however. 
enduring through time or 4-dimensional entities as having temporal parts and being extended through time. Both theories appear to answer the question of what persistence is equally well and it is not obvious how one should choose between them.

If one looks at the historical development of the above examples, the theories involved often start out as genuinely distinctive positions, but as they are adjusted to take objections into account and to better fit experiential and intuitive evidence, they begin to cover the same explanatory ground equally well. The process by which metaphysical theories are brought into harmony with each other has been called 'difference minimisation' by Karen Bennett (2009) and it leads to seemingly very different ontological theories matching each other in explanatory strength. Difference minimisation is not an inevitable process - some metaphysical theories remain as outliers which are not candidates to be treated as equivalent with any others - but the pull towards maximal explanatory power with minimal counterexamples does tend to encourage philosophers to produce theories which can each account for the same range of phenomena and this is where plurality appears.

The response to plurality has been extensive and varied. For some realist metaphysicians, the challenge is to determine which of the theories is the correct one and to argue its corner, or else to adopt an attitude of epistemic humility about the way the world is in the face of metaphysical stalemate. Others recommend an anti-realist or instrumentalist understanding of the categories and dependency relations which the respective theories postulate. One might, however, take the similarities between the theories to indicate metaphysical equivalence between them, at least in some important respects. But what would the implications of such equivalence be? Accepting that theories are equivalent might permit one to dismiss the debate as purely terminological - a move which is amenable to both realists and non-realists alike, since we are on this view just using different words to talk about the same things - or one might use this position to take a more robust anti-realist stance about the entities which our theories postulate. Alternatively, one might marshal this metaphysical equivalence between theories in defence of realism in order to argue for metaphysical structuralism: equivalence between metaphysical theories might allow us to overlook the specific ontological differences between different theories which explain the same phenomena in favour of concentrating upon the important relations which they determine between (for instance) similarity, causality, modality, meaning and so on. Such an account could turn out to be an ontologically satisfying way to preserve metaphysical realism, albeit realism about structures rather than the entities which they relate, and such a move would also capture the sense in which plurality 
does not seem to matter that much in metaphysics, even for realists, in the areas in which it occurs. Moreover, a metaphysical structuralist approach to realism would still be plausible if plurality of metaphysical theories turns out not to be a general metaphysical problem but one which only affects certain areas of enquiry. In cases where only one theory seems viable, we could either allow that there may be alternative equivalent theories which we have not yet formulated and give a structuralist account of the theory in question, or we might simply accept realism about the entities of that particular theory while retaining the emphasis on its relations with other theories.

\section{Dimensions of Difference}

\subsection{Metaphysical Equivalence}

So far, there has been rather loose talk of there being a plurality of metaphysical theories, and of metaphysical theories being equivalent to each other. But what does that amount to? Before we can embark upon a discussion of the plausibility of structuralism in metaphysics, we need to be more precise about what equivalence or near equivalence between metaphysical theories is. ('Near equivalence' is included in this discussion because one might want to accept that distinct metaphysical theories are all serious contenders to explain a certain phenomenon without making any philosophical or formal claims of equivalence between them; that is, one might accept that there is a problem of plurality and that the theories involved are in some sense interchangeable without accepting that the theories involved are equivalent. I will keep the notion of 'near equivalence' deliberately vague for that reason.)

Although we have, roughly speaking, distinct theories which characterise the same pretheoretical subject matter (such as the persistence of objects, or the nature of similarity and difference), the alternative theories involve complicated systems of ontological categories and dependency relations between them which do not correlate with the categories and relations postulated by other theories in any obvious way. There are, for instance, obvious differences between a theory which says that individual objects are bundles of tropes bound by a fundamental compresence relation and one which characterises them as being instantiations of universals inhering in a substratum. These two theories say different categories of entities exist which relate to each other in different ways. Yet, one might argue that these theories are equivalent to each other (Benovsky 2008), or else claim that the differences between the theories are irrelevant in such as 
way that accepting one rather than another is largely a matter of choice (Allen 2016, 97). On what grounds are such equivalence (or near equivalence) claims being made? Some clarification is in order.

First, it is important to note that there are ontological and epistemological conceptions of theoretical equivalence. On the ontological conception, there is a fact of the matter about whether theories are equivalent, whether or not we can determine that they are; in the manner of constitutive identity criteria for entities, there are equivalence criteria for theories. (One might want to call these constitutive equivalence criteria identity criteria, but I will opt not to do that in order to maintain some conceptual distance between equivalence and identity. One might, for instance, want to argue that there can be equivalent theories which are not identical because they utilise different terminology, or postulate different primitives or categories of entities, or have different internal structure.) On the epistemological conception, theories are equivalent to each other if more than one theory fits the evidence in the requisite way. One could draw an analogy between this epistemic conception of theoretical equivalence and Bas van Fraassen's (1980) account of scientific theories being 'empirically equivalent' to each other; that is, there being distinct theories which explain the observable evidence while disagreeing about which unobservables there are. Theories are empirically adequate when they 'save the phenomena'; that is, they account for the evidence just as well. But, in the context of metaphysics, what counts as evidence for a theory is another rather controversial matter. I will postpone discussion of that until the next section, and consequently delay discussion of the epistemic conception of metaphysical equivalence.

If we consider constitutive criteria, there are roughly speaking two ways in which the equivalence of metaphysical theories could be characterised: one might accept that theories (or entities, primitives and so on) are the same if they do the same thing, or one might distinguish them in virtue of their nature or content. With this in mind, we can borrow a distinction from Jiri Benovsky between different conceptions of ontology: the Content View (the conception of something according to its nature) and the Functional View (the conception of something according to what it does). Benovsky $(2013,346)$ draws this distinction between different conceptions of the primitives of a theory, entities which, he argues, are postulated as problem-solvers to fill explanatory gaps in a theory and which are not themselves open to analysis. Examples of such primitives include compresence (in trope theory) which binds tropes into particular objects, or some versions of the instantiation 'relation' in virtue of which instances of universals are exemplified by particular things. It seems plausible to say that compresence in trope theory and substratum in some 
accounts of universals and particulars are distinct on the content view, and yet coincide on the functional view; insofar as we care about what these primitives do, they are the same since they bind or coalesce whichever entities play the properties role (or their instances) into distinct particular individuals.

It seems plausible to suggest that we could extend the application of these two conceptions of equivalence from the primitives to other elements of the ontology, such as categories of entities and theories. Thus, for instance, we can think more broadly about the function of a theory as a whole (explaining similarity and difference, or persistence, or composition) and its content, or about the function or the nature of the entities and processes it involves. (We should emphasise, however, that the conceptions are not mutually exclusive: it may be that the nature of an entity is its function, in which case the Content View and the Functional View coincide. ${ }^{7}$ )

Within these broader categories of metaphysical function and content, one might have different views about what counts as sharing a function or what determines similarity of content, leading to differing accounts of how we should individuate ontology and consequently when it is permissible to treat a category or theory as equivalent or nearly equivalent. For instance, if we are interested in determining sameness of content, some might be happy with extensional equivalence, while others would require finer-grained intensional or hyperintensional individuation to underlie claims of equivalence. ${ }^{8}$ Clearly, the more restrictive the criterion of equivalence one accepts, the less likely it is that theories will turn out to be metaphysically equivalent to each other. For instance, those who accept a hyperintensional account of theoretical equivalence, such as theories being equivalent if they are true in all the same possible and impossible worlds ${ }^{9}$, are much less likely to encounter instances of plurality than those who advocate extensional equivalence, and consequently may not accept that cases of plurality ever occur. Those who accept intensional equivalence are more likely than the hyperintensionalists to encounter metaphysical equivalence, but the theories which turn out to be equivalent on their view may still be restricted to rather trivial cases (such as the translations of a French theory into English, for example ${ }^{10}$ ). Thus, it is unsurprising that the primary basis for most claims of metaphysical equivalence between theories - including those

7 For instance, this would be the case with accounts of properties or mental states which treat them as being entirely exhausted by their function. My view diverges from Benovsky's here because he defines 'content' in opposition to 'function' as 'a nature which is not functional' $(2013,345)$. I prefer to avoid this opposition if I can.

8 See, for instance, Miller 2017 which replaces her earlier view (2005b) that inter-translatability is sufficient for metaphysical equivalence.

9 I am using the talk of impossible worlds as a façon de parler, although one can try to characterise impossibility in modal realist terms (Yagisawa 1988).

10 There may be reasons to deny that theories translated between languages are content equivalent, but I will not pursue this matter here. 
discussed so far - is their functional equivalence, rather than equivalence of content: if two theories explain the same phenomena, or the entities, relations and primitives of the respective theories combine to produce the same effects (either explanatorily, or ontologically in terms of the entities they ground ${ }^{11}$ ), they count as equivalent on the functional view. Any differences in content between them are irrelevant to their explanatory power relative to a specific phenomenon.

We have to be careful here to relativise functional equivalence in this way to the explanation of a specific phenomenon or phenomena. Two theories are functionally metaphysically equivalent with respect to a specific explanandum (or set of explananda) if they do the 'same overall [explanatory] job’ but also, Benovsky adds ‘in the same way relevantly to an apt level of analysis' (2013, 351). These latter caveats are added in order to avoid very general equivalence claims, such as the equivalence of Locke's materialism and Berkeley's dualism (say) on the basis that there is only a difference in content between matter and spirit, but they add an awkward vagueness to the criterion which it might have been preferable to avoid. A reasonable level of detail is required for the equivalence claim to avoid triviality.

I will not pause to consider the merits of these different criteria here, since they are somewhat tangential to the discussion at hand and it is enough to acknowledge the difference that they will make to the existence of cases of plurality. Furthermore, in actual cases of metaphysical disagreement, the conception of equivalence in play is more often the epistemological one: there are cases in which metaphysicians arrive at competing theories on the basis of the same evidence and the same methodology; and given that, there are no grounds upon which one can make a principled choice between the theories.

\subsection{Metaphysical Evidence and Metaphysical Methodology}

In metaphysics, the relationship between theory and evidence is more complicated than in science (although I don't mean to suggest that the scientific case is straightforward), since there is a wide range of views both about what constitutes acceptable evidence and about which methods may be deployed to formulate a theory on the basis of that evidence. Following Anjan Chakravartty (2017, ch. 2 and ch. 3), we can talk about philosophers adopting different epistemic stances towards metaphysical theorising which involve commitments to types of evidence and methods and which can be plotted along something of a spectrum as a greater variety of evidence and methods are

11 I am using 'ground' in a very wide sense here to mean 'determine the existence of'. 
permitted. ${ }^{12}$ At one end of that spectrum, a strict logical empiricist will only accept the evidence of experience, and perhaps also mathematics and deductive (and, more controversially, inductive) logic. Those who are committed to naturalised metaphysics consider evidence and methodology to be legitimate in metaphysics if they are also legitimate in science ${ }^{13}$; they may also restrict what counts as a legitimate metaphysical explanandum to questions which arise specifically in science or which arise out of anomalies in scientific theories. (For instance, Ladyman and Ross 2007.) Further along the spectrum, others might accept common-sense (and other) intuitive data, substantive synthetic a priori claims and more 'relaxed' forms of reasoning including inference to the best explanation applied to metaphysical hypotheses (if this has not been admitted already), conceivability arguments, intuitive inclinations, or imaginative speculation. ${ }^{14}$ Theories which are indistinguishable with respect to one evidential basis may be distinguishable with respect to another: logical empiricists may agree that there is no basis upon which to choose between theories $\mathrm{T} 1$ and T2, while someone who accepts greater involvement of a priori reasoning in their metaphysics, or permits more ambitious inferences from the evidence might find a basis upon which they might be distinguished.

Thus, on its epistemic conception, it makes sense to talk about metaphysical equivalence only given an agreed epistemic stance concerning how metaphysical inferences should be made and justified. Philosophers engaged in a dispute need not accept each other's stances; but claims of metaphysical equivalence should be regarded as acceptable only relative to a particular epistemic stance. I will leave as an open question whether there is a 'most permissive' epistemic stance which encompasses all the evidence types and forms of justification included in the others. If this were the case, and if theories were equivalent relative to that over-arching 'super-stance' (as one might call it), then one might argue that on the epistemic conception they are metaphysically equivalent tout court.

In what follows, I will treat the epistemic conception of theoretical equivalence as being the one which matters from the point of view of formulating and comparing metaphysical theories. From the point of view of being able to comparatively evaluate the merits of respective theories, a point of difference between them cannot make a difference if we are unable to discern what that

12 Nothing in my argument will require the truth of Chakravartty's voluntarist claim that adopting one epistemic stance rather than another is a matter of choice.

13 Whether naturalised metaphysics is equivalent to logical empiricism will depend upon one's account of scientific evidence and methods, but most naturalised metaphysicians would consider their evidence base and methodology to be broader than that of most logical empiricists, at least because they have a direct realist conception of at least some empirical evidence and include inference to the best explanation as an acceptable form of reasoning.

14 One might also include assessment in terms of theoretical virtues. 
difference is. Metaphysical equivalence as I will be using it is therefore unashamedly an epistemic notion: theories are equivalent relative to a specific epistemic stance because utilising the methods and evidence which that stance allows does not permit our being able to rule one theory out in favour of the other. Therefore it is best to interpret the examples sketched in the first section as saying that given a shared epistemic stance (and thus a shared evidential basis), the theories considered are functionally equivalent. Furthermore, given that the examples are drawn from areas of metaphysics which are quite permissive about methods of metaphysical justification, the theories will remain equivalent according to all epistemic stances which accept fewer types of evidence and fewer methods (presuming, of course, that such theories can be formulated on the basis of those narrower stances).

But why choose functional equivalence as the relevant relation? Perhaps the metaphysical realist could insist upon content deciding between functionally equivalent theories. However, since content is by definition not playing a significant explanatory role, it is difficult to see how differences in content would permit a choice between theories to be made on the epistemic conception of equivalence under consideration. (Even if the evidence base includes intuitive inclinations towards ontological categories, this will not provide grounds for choice within a shared epistemic stance since such inclinations are an individual matter: someone might reject tropes because compresence seems incoherent to him, while someone else may have no difficulty in including tropes in her favoured ontology.)

Plurality of metaphysical theories begins as an epistemic problem, arising when the epistemic resources to decide between theories run out, but it is not inevitably an ontological one. From a realist perspective, although we cannot determine which theory is the correct one, it may be the case that the epistemically equivalent theories are not ontologically equivalent: there may be a correct theory, but we will never know (or be justified in believing) which theory this is. Realists still have two options: they could accept a version of Kantian humility with respect to which metaphysical theory is the correct one and what we can justifiably believe about the nature of the world $^{15}$; or they could simply be ambivalent about the problem and accept that somehow the categories and structures of any and all of the theories somehow latch onto whichever entities and processes constitute the world. ${ }^{16}$

15 This attitude is typified by Lewis 2009.

16 This option was suggested by Richard Woodward (in conversation) and is subtly different from humility in that it takes a relaxed view of the relationship between theories and the world. It does not permit strict reference relations between predicates and the entities which the world contains: we can be realists about any of the theories. 
However, these options are unlikely to be attractive to ontological realists since it is a consequence of accepting them that we cannot take our metaphysical theories literally any more; metaphysical theories do not tell us what the mind-independent world is like in a robust sense. Acceptance of a perdurantist account of persistence does not, for instance, tell us that objects do persist by perduring, nor does a mereological universalist theory of composition tell us that there really is an object composed of my computer and my desk. This is an unsatisfactory result for ontological realists since they would have to relinquish their commitment to the claim that our theories literally tell us which categories of entities and processes there are (to a suitable degree of accuracy) in favour of accounts which either treat the nature and structure of the world as noumenal or which accept that once we have exhausted the means to decide between theories, any theory tells us how the world is. ${ }^{17}$ If one cares about this, neither humility nor ambivalence are the right attitudes to adopt to the plurality problem.

\section{Metaphysical Structuralism and its Limits}

The foregoing discussion clarifies, to some extent, the conditions required for metaphysical equivalence between theories and what is involved. One might, at this point, recommend that realism be abandoned in favour of some form of pragmatism or anti-realism, accepting that there is a point when, if we have used up all weapons in our epistemic armoury and still have a plurality of theories available, the ontology which they postulate cannot be treated realistically. ${ }^{18}$ But now we are armed with a clearer account of how metaphysical equivalence could work on a local level by taking the functional view of theories, it is tempting to extend this success to metaphysics more generally. Before the realist abandons his own position, he should at least explore the potential of applying a structuralist approach to metaphysics itself. It is to this project and the difficulties which arise that I will now turn.

17 Two further suggestions have recently been made to reconcile metaphysical plurality with realism: perspectivism (Agazzi 2016; Saatsi (forthcoming); Massimi and McCoy (forthcoming)) and dispositionalism (Chakravartty 2017, 194-200). But both fail when applied to the cases under discussion, since they presuppose that the difference in theoretical content comes about as a result of different contexts, even though Chakravartty argues that the truth of the ontological explanation transcends that context. In the cases of plurality above, the plurality arises and the different theories are applicable across all the same contexts and so we cannot argue that an apparent difference in theoretical content is a matter of perspective, or that it arises due to the existence of dispositions which manifest in some contexts and not others (but are present (although not always manifested) across all contexts). There is more to be said on this point and I aim to take it up in later work.

18 See, for instance, Benovsky (2016) who uses the aesthetic properties of theories as a deciding factor between them, thereby giving an anti-realist account of metaphysics. 
The thinking behind metaphysical structuralism is as follows: we want a metaphysical account of similarity and difference (for example) and we have several functionally equivalent theories. Why (for realists) should multiple successes count as failure from a theoretical point of view? Furthermore, since the theories are functionally equivalent, one could be used in place of another and there would (by definition) be no noticeable difference, except in the internal structure of the theory which is primarily of interest to metaphysicians of properties. These theories answer the question of what a property is just as well as each other and they provide an account of the way properties relate to other categories of entities just as well as each other too. Each one can be used as the basis of theories of kinds, laws of nature, modality, meaning, types of objects, and in theories of other phenomena in which properties are relevant. (The resulting theories of these phenomena may be different for the respective ontological accounts of properties, but nevertheless explanations are available.) The situation is similar, one might add, to those in mathematics in which different set theoretic axiom systems yield equivalent results. Moreover, one can characterise the ordinals settheoretically using von Neumann's system such that each ordinal is the well-ordered set of the smaller ordinals, or in terms of the Zermelo-Frankel system where each ordinal is simply the set of the ordinal directly preceding it. ${ }^{19}$ For mathematical purposes, these make no difference and the choice between them is arbitrary. We can extend this observation to less obviously equivalent ontological accounts of the natural numbers as well such as those which treat numbers as abstract objects, formal symbols, or as mind-dependent entities such as ideas. What matters is not the nature of mathematical entities themselves but their relations to other mathematical entities. Furthermore, we might argue on this basis that mathematical structure exists prior to the entities which play a role in that structure; that is, the structure is ante rem rather than in re. If we accept that the same is true in metaphysics and we can apply this analysis to the difference between trope theory and the theory of immanent universals, or resemblance nominalism, then another long-standing ontological dispute is allayed. To generalise the point, what we are trying to capture with metaphysics are the relations between different metaphysical phenomena such as similarity, modality, meaning, composition and so on, the structure into which they fit; and we can construe this structure realistically even when we have more than one adequate account of the categories and processes which occupy the roles fixed by the nodes of that structure. On this proposal, we could then build upon local functional equivalence between theories to develop a broader metaphysical worldview, minimising ontological disagreement along the way. That is the metaphysical structuralist's aim. But does it stand up to

19 Von Neumann's version of the ordinals gives $\varnothing,\{\varnothing\},\{\varnothing,\{\varnothing\}\},\{\varnothing,\{\varnothing\},\{\varnothing,\{\varnothing\}\}\},\{\varnothing,\{\varnothing\},\{\varnothing,\{\varnothing\}\},\{\varnothing$, $\{\varnothing\},\{\varnothing,\{\varnothing\}\}\}\}$ and so on; Zermelo-Frankel gives $\varnothing,\{\varnothing\},\{\{\varnothing\}\},\{\{\{\varnothing\}\}\},\{\{\{\{\varnothing\}\}\}\}$ and so on. 
scrutiny? I will discuss four main weaknesses with the project of generalising the notion of local metaphysical equivalence between theories to produce a metaphysical structuralist account of the metaphysics of the world.

\subsection{Primitives}

The first problem arises even when we restrict ourselves to considering the local functional equivalence of theories. The difficulty here is that apparently functionally equivalent primitives or entities might, in some areas as yet undiscovered, behave in different ways to each other. For instance, Benovsky considers whether there is a difficulty about maintaining functional equivalence between the theoretical primitives compresence and substrata on the basis that only the latter are able to exist independently. He concludes that there is not $(2009,2013)$ on the basis that this feature of substrata has no explanatory relevance to the theory of immanent universals and does not affect the functional equivalence between that theory and trope theory insofar as they provide an account of kinds of objects. The difference here is one of content, rather than function. Nevertheless, Benovsky concludes that cases such as this require us to acknowledge that equivalence claims might only be partial (2013, 350): the possibility that apparently functionally equivalent ontology could diverge remains and it is a possibility which keeps the equivalence claim applicable only within a specific explanatory domain where we can trace respective functional roles to make sure that they coincide.

In principle, one might think that this could be done and is essentially an epistemic matter. For instance, in the example of the equivalence of trope theory and universals theory in the formation of particulars (which is founded upon the functional equivalence between compresence and substrata), one might think it unlikely that there is something that compresence can do which a substratum cannot which would not turn out to be a trivial difference, in the sense it would not make a difference to any other theories in which these primitives appear. But it is still an open possibility that functions could diverge and so as the scope of equivalence is broadened to include more theoretical structure, we need to distinguish cases of functional equivalence on a domain of enquiry from functional equivalence tout court. This project seems manageable when we consider primitives, since primitives are often ontologically quite ‘thin': they are postulated to do a specific job, to play a specific explanatory role, and do not conceal a hidden nature which might turn out to possess hidden functional potential. They are usually, also, internal to the theory in which they 
appear and do not have implications outside it. Nevertheless, concerns about the functional potential of primitives being 'hidden' on a local level bring to light some related, but more serious, concerns about other implicit aspects of theories which might prevent widening the scope of claims of metaphysical equivalence.

\subsection{Presuppositions}

Chief among these wider concerns is the fact that metaphysical theories involve commitment to presuppositions without which they would not be candidates to be viable metaphysical theories at all. Furthermore, these presuppositions concern areas of metaphysical interest outside the local explanatory focus of the theory in which they are used and these areas often, but not always, vary between theories about the same phenomenon. This can be illustrated by considering metaphysical accounts of the ontology of similarity and difference. For example, ontological commitment to transcendent universals involves commitment to the existence of abstract objects (abstract in the sense of their being objects which are neither spatial nor temporal), while the more plausible versions of class and resemblance nominalism require commitment to modal realism if they are to avoid the coextension problem. (Armstrong 1989, 50-1; Rodriguez-Pereyra 2002, ch. 5) It seems that although the theory of transcendent universals and that of resemblance nominalism are metaphysically equivalent on a local level, in the sense that they both adequately explain objective similarity and difference, the acceptance of one theory rather than another implicitly commits one to specific metaphysical claims which a holder of the other theory might want to avoid. Not that, in this case, the presuppositions are in contradiction with each other, although one might want to be a resemblance nominalist precisely to avoid ontological commitment to abstract objects; while, on the other hand, someone else might want to avoid resemblance nominalism on the grounds that it requires commitment to modal realism.

According to Stewart Shapiro (1997, ch. 7 especially 228-9), another example of this can be found in structuralist theories in the philosophy of mathematics. In re structuralism in which the existence of patterns of existing objects determine the existence of structures requires the existence of sufficiently many objects to ground the structures of mathematics. It achieves this with ontological commitment to primitive logical modality which governs what could exist (it could resort to commitment modal realism at this point, but that would be a less popular and more obscure option). On the other hand, ante rem structuralism is committed to the existence of structures, 
whether or not objects exist to instantiate those structures. This involves presupposing the existence of abstract entities again, transcendental structures which can exist uninstantiated and which form the ontological basis of mathematics.

The problem of presuppositions is both different from and more intractable than the question of whether functionally equivalent theories imply different versions of a theory of a related phenomenon (when, for instance, different property theories imply different accounts of natural laws). In this latter case, the resultant theories may differ without this having any further implications for the overall metaphysical structure: there are simply alternative, yet functionally equivalent, theories explaining laws of nature, without this having a knock-on effect of metaphysical explanation beyond that. On the other hand, if the presuppositions required by two theories differ, then they are bound to fit into distinct metaphysical structures.

To put the difficulty here in general terms, theories T1 and T2 are functionally metaphysically equivalent with respect to specific local explananda, but because of the respective presuppositions P1 and P2 required to sustain T1 and T2, the wider structure in which T1 fits will be different from the wider structure in which T2 is embedded. Details which were functionally irrelevant on a local level, such as commitment to the existence of abstract objects, or primitive modality, or to possible worlds existing in the same sense as the actual one, are relevant when the wider metaphysical context of the theories in which they appear is considered. Unlike the case of primitives, these presuppositions are substantive, involving commitment to robust categories of entities or systems, and they have implications beyond the original theories for which they are presupposed. Different broader metaphysical structures may be determined by locally functionally equivalent metaphysical theories; plurality has reappeared at the level of structure and so it has not been successfully dealt with by shifting to a structural understanding of metaphysics.

One might suggest two responses to alleviate the severity of this objection. First, one might attempt to minimise or to explain away the distinctive contributions which the locally functionally equivalent theories make to the broader metaphysical structure by drawing an analogy between metaphysical structuralism and structuralism in mathematics. In the mathematical case, the different functionally equivalent accounts of number differ significantly in certain respects. For instance, as Benacerraf points out, the different set theoretic conceptions of number work just as well as each other and yet the Zermelo-Frankel conception is different from that generated by von Neumann's axioms, and therefore different facts are true of the individual numbers. For example, on the latter, but not the former, it is true that three is a member of 17 . Furthermore, if we examine other 
conceptions of number amenable to the structuralist (on the basis that they produce functionally equivalent mathematical structures) further differences occur: the Fregean account of natural numbers considers them to be abstract objects and so, as in the case of the theory of transcendent universals above, accepting Frege's account of numbers involves presupposing the existence of abstracta, whereas accepting a formalist or set theoretic account of number may not. But, in mathematical structuralism, these differences are not cause for concern; it is precisely because the functional roles of the numbers are all that is important to mathematics (if you are a structuralist) that the difference in the presuppositions embedded in a Fregean account and a set-theoretic account of numbers is not relevant and we can simply account for mathematics in terms of structure. ${ }^{20}$

Can we say that the same is true about presuppositions when we try to formulate metaphysics structurally? On a local level, it seems that we can agree with the tactics of the mathematical structuralists: the transcendent universals' theorists' commitment to the existence of abstract objects makes no difference to the account of similarity and difference in comparison with other theories of similarity and difference which differ in content and do not include that presupposition. But, as we begin to expand the metaphysical structure to include novel explanatory questions, the prior commitment to abstract objects places constraints on the structures in which the theory of transcendent universals can appear. The key move in the philosophy of mathematics was to point out that differences in content are not mathematically relevant, but a similar move cannot be made here. We cannot say that existential assumptions about the existence of certain categories of entities, or specific accounts of modality and so on are irrelevant to metaphysics because they are also part of metaphysics. There is no convenient 'extra' area of discourse in which to corral differences in theoretical content which have effects outside the local theory in order to get on with developing metaphysics. (It is worth noting that the mathematically irrelevant differences between accounts of number in mathematical structuralism (discussed above) are metaphysically relevant too.) In some cases, when we begin to consider the broader metaphysical picture, locally functionally irrelevant details matter, and their broader relevance to the respective metaphysical systems of theories in which they are embedded cannot be easily dismissed. The analogy with mathematical structuralism does not hold.

20 I am not advocating mathematical structuralism here nor intending to argue for it. The issues here are whether metaphysical structuralism is as plausible as mathematical structuralism, and whether metaphysical structuralism can respond to certain objections in an analogous way to mathematical structuralism. 
Second, one might suggest that the fact that $\mathrm{T} 1+\mathrm{P} 1$ implies structure $\mathrm{S} 1$ and $\mathrm{T} 2+\mathrm{P} 2$ implies structure S2 does not matter because, if we expand the metaphysics far enough, there may be an overarching structure which captures both variants and within which they are functionally equivalent again, both locally (in certain respects) and globally. This overarching structure could sustain realism and again make plurality a trivial problem. But although this response may turn out to be viable in some cases, it is prima facie implausible if one hopes to use metaphysical structuralism to sustain realism. First: why would we have reason to believe that such an overarching structure exists? To find it credible, it seems likely that one would have to presuppose the existence of a metaphysical structure to the world, as one would no longer have good reason to believe that there is one on the basis of theories already formulated; and this move would be begging the question in favour of realism. Thus, realism remains a coherent position to hold, but one has to presuppose realism to sustain it. Second, since plurality has now appeared at two levels of metaphysics: at the level of first order theories explaining local phenomena and at the level of the structures in which those theories stand when functionally defined, there is no reason to believe that plurality will not strike again at the level of the over-arching structure. Once again, presupposing realism to escape this worry is an unappealing prospect and seems rather ad hoc. If one has realist inclinations in metaphysics, there are simpler and less ontologically committed ways to presuppose that one's position is true.

\subsection{Holism}

The difficulty in 3.2 that functionally equivalent theories T1 and T2 may respectively conceal distinct presuppositions P1 and P2 which have implications more broadly than the theory at hand has a counterpart which underscores quite how holistic metaphysical systems can be. This difficulty concerns the subject matter of the presuppositions themselves as these too may involve commitment to a certain type of theory; that is, theory T1 requires the truth of presupposition P1, but P1 is itself a disguised commitment to any one of a group of functionally equivalent theories. For instance, if $\mathrm{T} 1$ is resemblance nominalism, then P1 could be modal realism (which, as noted above, class and resemblance nominalism both require in order to circumvent the coextension problem). But there are several different theoretical variants of modal realism, not to mention some other theories of modality which reify possible objects in a way which would be consistent with class and resemblance nominalism's requirements that possible members of classes exist in the same sense as 
actual ones. Thus, in this case, we have a situation in which the presupposition required by a theory can be grounded in any one of a cluster of theories which take a realist attitude to possibilia. ${ }^{21}$ While, for the purposes of underscoring class or resemblance nominalism, these theories are functionally equivalent to each other, and (perhaps) they may also be regarded as being so for the explanation of modality, they will each involve presuppositions of their own which, as described in 3.2, will affect the broader metaphysical structures within which each theory can be a part. For instance, a theory which reifies possible worlds which are spatio-temporally isolated from the actual one will occupy a different structure from a theory which reifies modal dimensions in the one actual world alongside temporal and spatial dimensions.

This kind of phenomenon serves to illustrate a knock-on effect of how aspects of a theory which are locally functionally irrelevant to the theory (and which serve only to mark differences in content between it and other functionally equivalent theories) can affect the broader metaphysical system within which a particular theory is embedded. A theory can have non-local implications which it does not share with other locally functionally equivalent theories due to the presuppositions it requires; and in turn the theories in which these implications are true have their own respective non-local implications; and so on to more theories. Theories which are functionally equivalent with respect to the answers which they give to a narrow range of metaphysical questions quickly cease to be so when the specific ontological assumptions which ensure their coherence are considered. But the knock-on effect comes into play when these assumptions are each considered in their theoretical context, where we would expect the problem of non-local implications to recur.

Given the potential chains of implications which I have set up, it also seems possible that the presuppositions of theories could ultimately affect each other, either directly or through a circle of implications. For instance, let us presume that the presuppositions of a theory T3 require theory T4, while those of T4 require T3. Because the circularity is ontological and not epistemological, the existence of a circle does not matter in itself, but here we have a genuine case of ontological interdependence: T3 requires the ontology of T4 and T4 requires the ontology of T3. Although T3 and T4 might each be locally functionally equivalent to a range of respective alternative theories, they can only belong in a larger system with each other; attempts to widen the scope of the equivalence further would fail. One fairly simple example of such circularity can be found in certain

21 I will not investigate whether there are any broadly speaking modal realist theories which would be problematic from the point of view of resemblance nominalism, since it would be rather tangential to the current discussion. Nevertheless, one might think that a theory which reifies a very wide range of possible worlds, including those with non-standard logics and even impossibilia, would not be compatible with the role for which nominalists invoke a modal realist account of possibilia (to maintain extensional identity conditions for classes). 
accounts of concrete particular objects and events as being essentially spatio-temporally identified and individuated, a theory of particulars which thereby requires the existence of space and time or space-time. Although one need not be committed to a specific account of space and time to make this work, one might opt for a reductionist account of space and time in which space and time are entirely reducible to matter and change, which very quickly requires the existence of particular objects and events. This example of an ontological circle is a small one and could be avoided by taking on alternative ontological commitments such as a substantival conception of space and time, or a different account of the identity and individuation of concrete particulars, but it is nevertheless a coherent possible theoretical position to maintain and it makes two theories interdependent in virtue of the ontological presuppositions which they involve. ${ }^{22}$

Perhaps one could one get around this problem. Could we choose a theory functionally equivalent with T3 (let us call it $\mathrm{T}^{*} 3$ ) and conjoin it with a theory functionally equivalent with T4 (let us call this $\left.\mathrm{T}^{*} 4\right)$ )? Is the conjunction of $\mathrm{T} * 3 \& \mathrm{~T} * 4$ functionally equivalent with $\mathrm{T} 3 \& \mathrm{~T} 4$ ? In terms of what these theories do, it seems the answer has to be 'yes', except when we consider the dependency relations which they postulate between the specific ontological categories which they employ. In this matter, T3\&T4 involves internal dependency relations which $\mathrm{T} * 3 \& \mathrm{~T} * 4$ does not, and one might feel that some significant metaphysical explanation is lost with the latter theory. After all, one might feel that one of the most important questions in metaphysics concerns such dependency relations, or to put it in Schaffer's terms, 'what grounds what' (2009). On the other hand, one might be prepared to overlook the loss of this internal explanation and treat T3\&T4 and $\mathrm{T}^{*} 3 \& \mathrm{~T} * 4$ as functionally equivalent with respect to certain metaphysical questions. Nevertheless, one would still have to tread carefully in expanding metaphysical theories into broader theoretical structures and this cannot be done on the basis of their respective local functional equivalence alone: one could not, for example, conjoin $\mathrm{T} 3$ with $\mathrm{T} * 4$, rather than $\mathrm{T} * 3$, since $\mathrm{T} 3$ would be inadequate without T4, lacking the truthmakers for a presupposition which makes T3 coherent.

These observations are unlikely to be news to metaphysicians who have thought about system-building in metaphysics in any detail, since when one tries to construct comprehensive metaphysical systems, rather than simply readjust local areas of theory, the extent to which the background of one theory implies another, which implies another (and so on) soon becomes obvious. What seem to be functionally equivalent theories on a local level, with respect to a

22 I will continue to count the theories as two in the course of this discussion because they aim to explain different (and separable) metaphysical phenomena: particulars and space and time. I accept that once we are aware of such situations, one might prefer to consider the theories to be one larger theory, especially given what I have to say in the next paragraph. 
restricted number of metaphysical problems, are, on further inspection, embedded in very different metaphysical systems.

The conclusions we should draw from this are that, local functional equivalences notwithstanding, metaphysical systems are frequently (if not always) holistic and that we cannot properly think of local theories as if they occupy nodes in an overarching structure because we cannot swap one theory for a locally functionally equivalent one without potentially changing the 'shape' of the structure concerned. Functional equivalence of a theory within a structure should be a formal matter - that is, a theory occupies the place it does only in virtue of the relations it bears to other parts of the metaphysical system (that is, what it is functionally equivalent to) - but these examples show that this is not the case. In part, this problem arises with metaphysical structuralism, and not mathematical structuralism, because the latter can corral the locally functionally irrelevant content differences between functionally equivalent theories (which sometimes become functional differences on a larger scale) and treat them as metaphysically relevant but mathematically irrelevant and so as being irrelevant to the larger structure. Metaphysical structuralism does not have this option: everything in the theory is metaphysics and so it is, by definition, relevant to the structure which results from connecting theories together. As we synthesise theories which explain different areas of metaphysical interest, the result is not one structure but many, although that does not prevent our noting functional equivalences at each stage of the synthesis. Furthermore, theories which are functionally equivalent to each other on one scale are not functionally equivalent on another.

\subsection{What is metaphysical structure?}

Even if there are cases where the problems discussed in 3.1-3.3 do not arise, such that we find that there are structures in which groups of functionally equivalent theories stand in stable relations to other groups of functionally equivalent theories ${ }^{23}$, metaphysical structuralism faces a fourth problem: that it generates as many metaphysical problems as it solves.

Metaphysical structuralism was motivated in part by the promise of a mechanism which could retain realism in the face of plurality, removing the emphasis from the different ontological accounts provided by functionally equivalent theories in favour of seeing them as part of an overarching metaphysically explanatory structure. However, the advantages of this ontological

23 This may be because they share presuppositions about ontology external to the theory, or they only rely on very minimal extra-theoretical ontological assumptions. 
simplification are soon lost once we ask the rather obvious question of what the structure in question is. The answer to this question is likely to yield a resurgence of the very ontological disagreements which the adoption of structuralism was intended to solve, and consequently to reproduce the same problem of plurality. ${ }^{24}$

In response to this, one could decry interest in such questions and insist that metaphysical explanation can be brought to a halt at some point. But this move seems quite disingenuous when there appear to be legitimate metaphysical questions about why theories fit in the structures which they do and what those structures are, whether the relations between theories are explanatory, or something else (and if so, what that something else is), or whether they are explanatory and something else. Perhaps the dismissive attitude could be justified if we insist on the importance of understanding the similarities between first order metaphysical theories in order to put them to explanatory use and leave the discussion there. However, this strategy seems more pragmatic than realist in its motivation and is unlikely to satisfy realist metaphysicians.

Moreover, if one is defending structuralism in order to sustain realism, it seems difficult to deny that, if one is a structuralist, there is a structure there to be investigated. Refusing to ask further metaphysical questions does not seem acceptable from the realist point of view, regardless of whether this will lead to further difficulties with plurality. However, if we are permitted to deal with these problems with a plurality of structures by a further application of structuralism, there is some hope of stalling the burgeoning ontological disagreement at an early stage: along these lines, one might argue that if the different accounts of what a structure is are functionally equivalent to each other, then the differences in content do not matter. The plurality of accounts of the nature of structure would thereby be neutralised if we permit structuralism to be applied to the different explanations of structures themselves, and a potential requirement for more explanation (of the structure which explains the structures) could be avoided on the grounds that this is simply a demand for more of the same. We do not, if we adopt this strategy, halt the call for explanations in an ad hoc way, but we do establish that further explanations will not reveal anything interesting or novel.

\section{Conclusions}

24 Questions about the nature of structures have already been extensively discussed in the philosophy of mathematics and so I will not consider the details of the accounts which might be offered here. See, for instance, Resnik 1988, 1997; Hellman 1989, 1996, 2001; Shapiro 1997. 
In this paper I have argued that the local functional equivalence of metaphysical theories is not sufficient to fit them into a broader system because locally functionally equivalent theories make presuppositions which, while functionally irrelevant at local level (i.e. part of the content), have distal effects on which other theories they can combine with. This implies that functional equivalence is indeed domain relative and that we cannot swap one functionally equivalent theory for another with the guarantee that the system will be preserved. This emphasises the holistic (or partially holistic) nature of metaphysical systems with the implication that we cannot take a structuralist view of the metaphysics of the world in order to sustain realism in the light of metaphysical plurality. Frequently, functionally equivalent theories belong in different systems, determined by the primitive assumptions which they involve, and one cannot resolve the differences between these systems without presupposing that there is a single pre-existing metaphysical structure of the world into which they fit. Realism can be sustained, but only on the assumption that it is true, and so metaphysical structuralism does not resolve the plurality problem unless we are already assured that there is a grand over-arching structure into which our theories fit.

In the grander scheme of things metaphysical structuralism does not provide a general solution to the plurality problem for the realist because theories do not combine in the way in which one might have hoped that they would. In contrast to structuralism in mathematics and (arguably) in science, we are not capturing some over-arching, pre-existing structure with our theorising, but trying to construct explanations via the postulation of systems which are ultimately holistic in nature. What does this tell us about the nature of metaphysics? If plurality threatens realism, and structuralism cannot save it, then perhaps the best interpretation is some form of instrumentalism or anti-realism about the entities and relations between them which our theories involve. However, the other suggested realist solutions of humility and ambivalence remain on the table.

These observations do not harm findings of functional equivalence between theories at a local level, however. We can still talk about the functional equivalence of theories of similarity and difference, or particular object formation, or persistence, and so on. Nor does it prevent our combining functionally equivalent theories which explain one domain with theories which explain another; although each stage of combination involves taking note of the theories' content in addition to their functional role. We can only combine and assess theories into larger, more explanatory, ones in a piecemeal way by doing metaphysics at every stage. Although there are explanatory gains to be 
had from noticing the functional equivalence of theories, this move is not a methodological easy way out. ${ }^{25}$

\section{References}

Agazzi, Evandro. 2016. Scientific Realism Within Perspectivism and Perspectivism Within Scientific Realism. Axiomathes 26 (4): 349-365.

Allen, S. R. 2012. What matters in (naturalized) metaphysics? Essays in Philosophy 13: 211-41.

Allen, S. R. 2016 A Critical Introduction to Properties. Bloomsbury.

Armstrong, D. M. 1989. Universals: An Opinionated Introduction. Boulder, CO: Westview Press.

Armstrong, D. M. 1997. Properties. In D. H. Mellor \& Alex Oliver (eds.), Properties. Oxford:

Oxford University Press.

Benacerraf, Paul.1965. What numbers could not be. Philosophical Review 74 (1): 47-73.

Benacerraf, Paul. 1973. Mathematical truth. Journal of Philosophy 70 (19): 661-679.

Bennett, Karen. 2009. Composition, colocation, and metaontology. In David John Chalmers, David Manley \& Ryan Wasserman (eds.), Metametaphysics: New Essays on the Foundations of Ontology. Oxford: Oxford University Press: 38-76.

Benovsky, Jiri. 2008. The bundle theory and the substratum theory: deadly enemies or twin brothers? Philosophical Studies 141 (2): 175-190.

Benovsky, Jiri. 2009. The Self: a Humean bundle and/or a Cartesian substance? European Journal of Analytic Philosophy 5: 7- 19.

Benovsky, Jiri. 2013. Primitiveness, Metaontology, and Explanatory Power. Dialogue 52 (2): 341358.

Benovsky, Jiri. 2016. Meta-Metaphysics. Dordrecht: Springer Verlag.

Chakravartty, Anjan. 2017. Scientific Ontology: Integrating Naturalized Metaphysics and Voluntarist Epistemology. Oxford: Oxford University Press.

Chalmers, D., Manley, D. \& Wasserman, R. (eds.) 2009. Metametaphysics: New Essays on the Foundations of Ontology. Oxford: Oxford University Press.

25 I would like to thank the audience and organisers at the University of Düsseldorf Conference 'What do we do when we do metaphysics?’, especially Jiri Benovsky, Markus Schrenk and Richard Woodward, for criticisms and comments on an earlier version of this paper. 
Hellman, Geoffrey. 1989. Mathematics Without Numbers: Towards a Modal-Structural Interpretation. Oxford University Press.

Hellman, Geoffrey. 1996. Structuralism without structures. Philosophia Mathematica 4 (2): 100123.

Hellman, Geoffrey. 2001. Three varieties of mathematical structuralism. Philosophia Mathematica 9 (2): 184-211.

Hirsch, Eli. 1993. Dividing Reality. Oxford: Oxford University Press.

Hirsch, Eli. 2005. Physical-Object Ontology, Verbal Disputes, and Common Sense. Philosophy and Phenomenological Research 70 (1): 67-97.

Hirsch, Eli (2009). Ontology and alternative languages. In David John Chalmers, David Manley \& Ryan Wasserman (eds.), Metametaphysics: New Essays on the Foundations of Ontology. Oxford University Press: 231-58.

Ladyman, James and Ross, D., with J. Collier and D. Spurrett. 2007. Every Thing Must Go. Oxford: Oxford University Press.

Lewis, David. 2009. Ramseyan humility. In David Braddon-Mitchell \& Robert Nola (eds.), Conceptual Analysis and Philosophical Naturalism. MIT Press: 203-222.

Massimi, Michela \& McCoy, C. D. (eds.) (forthcoming). Understanding Perspectivism: Scientific Challenges and Methodological Prospects. New York: Routledge.

McCall, Storrs \& Lowe, E. J. 2006. The 3d/4d controversy: A storm in a teacup. Noûs 40: 570-578.

Miller, Kristie. 2005a. The Metaphysical Equivalence Of Three And Four Dimensionalism. Erkenntnis 62 (1): 91-117.

Miller, Kristie. 2005b. What is Metaphysical Equivalence? Philosophical Papers 34: 45-74.

Miller, Kristie. 2017. A Hyperintensional Account of Metaphysical Equivalence. Philosophical Quarterly 67: 772-793.

Resnik, Michael D. 1988. Mathematics from the Structural Point of View in Philosophie des Mathématiques. Revue Internationale de Philosophie 42 (167): 400-424.

Resnik, Michael D. 1997. Mathematics as a Science of Patterns. New York: Oxford University Press.

Rodriguez-Pereyra, G. 2002. Resemblance Nominalism. Oxford: Oxford University Press.

Rosen, Gideon \& Dorr, Cian. 2002. Composition as a fiction. In Richard Gale (ed.), The Blackwell Companion to Metaphysics. Oxford: Blackwell: 151-174. 
Saatsi, Juha (forthcoming). Realism and Explanatory Perspectivism. In Michela Massimi \& C. D. McCoy (eds.), Understanding Perspectivism: Scientific Challenges and Methodological Prospects. New York: Routledge.

Schaffer, Jonathan. 2009. On what grounds what. In David Manley, David J. Chalmers \& Ryan Wasserman (eds.), Metametaphysics: New Essays on the Foundations of Ontology. Oxford: Oxford University Press: 347-383.

Shapiro, Stewart. 1997. Philosophy of Mathematics: Structure and Ontology. Oxford: Oxford University Press.

Tahko, Tuomas E. 2015. An Introduction to Metametaphysics. Cambridge: Cambridge University Press.

Van Fraassen, Bas. 1980. The Scientific Image. Oxford: Clarendon Press.

Worrall, John. 1989. Structural realism: The best of both worlds? Dialectica 43 (1-2): 99-124.

Yagisawa, T. 1988. Beyond Possible Worlds. Philosophical Studies 53: 175-204. 